\title{
Limits to Early Mental State Reasoning: Fourteen- to 15-Month-Old Infants Appreciate Whether Others Can See Objects, But Not Others' Experiences of Objects
}

\author{
Brandon Woo (bmwoo@g.harvard.edu) and Elizabeth Spelke (spelke@wjh.harvard.edu) \\ Department of Psychology, Harvard University, Cambridge, MA 02138 \\ Center for Brains, Minds, and Machines, Cambridge, MA 02139
}

\begin{abstract}
Research provides evidence that infants infer what others can and cannot see from their differing perspectives, but do infants appreciate that their own perspective on an object can differ from that of a person who views the same object from a different direction? First, infants were shown two faces with screens in front of or behind them. Infants correctly inferred that a face that was visible to them was occluded to an actor sitting across from them. Then two experiments presented infants with two unscreened faces: one upright and one inverted. Infants attributed their own perspective on those faces to the actor: They inferred that faces that were upright to them were also upright to the actor across from them. Thus, infants appreciate that others may see things that they do not, but they fail to grasp that others may experience the same visible objects differently than they do.
\end{abstract}

Keywords: infancy; visual perspective-taking; theory of mind; social cognitive development

\section{Introduction}

The world can look different to different people, depending on their visual perspectives: What looks upright to me might look upside-down to you. In our social interactions, we consider not only what other people can see, but how the world looks to them. Here, we ask whether infants appreciate when someone else's perspective on a picture differs from their own.

\section{Understanding Whether Others See Objects}

A large and growing body of research has demonstrated that infants can reason about whether others can see an object or event, when others' perceptual access to the object or event differs from their own (e.g., Luo \& Johnson, 2009; Hamlin et al., 2013; Woo et al., 2017; see also, Phillips et al., 2020). Even when infants can see every object in a scene, they appreciate when others cannot. Moreover, some studies in this literature suggest that infants can reason about mental states that differ from what infants themselves know of reality (e.g., Onishi \& Baillargeon, 2005; Southgate et al., 2007; Woo \& Spelke, 2020; cf. Poulin-Dubois et al., 2018).

To establish agents' mental states, past research has manipulated what events and (parts of) objects could or could not be seen from another person's perspective. Yet, the perspectives that adults and older children can represent extend beyond what people can or cannot see. For example, by 4 years of age, children demonstrate an understanding in verbal tasks that the same object (e.g., a turtle) will look different to people who view it in different orientations (Masangkay et al., 1974). To our knowledge, no experiment has tested whether infants understand that people may look at the same object for the first time but see different things.

\section{Understanding Others' Experiences of Objects}

One possibility is that infants may be egocentric when challenged with a conflict between their own experience of an object and the experience of another person. Three-yearold children, who correctly answer questions about what others can and cannot see (e.g., Flavell et al., 1981; Flavell et al., 1978; Masangkay et al., 1974), struggle to reason about how a particular picture looks (e.g., upright or inverted) from the perspective of someone facing them. Children are apt to focus on their own perspective on the picture, thinking that what is upright to them is upright to others, regardless of others' perspectives (Birch \& Bloom, 2004; Flavell et al., 1981; Masangkay et al., 1974). Even as adults, people more readily consider whether others can see the same object than whether they experience a visible object in the same way (Surtees et al., 2012). If infants are egocentric, like 3-yearold children, then they may not appreciate the different ways that two people may experience the objects that both see.

Alternatively, infants may have an implicit understanding that others can experience objects differently than they do, despite young children's failure to express this understanding in explicit, verbal tasks. In situations involving no other agents, infants in the first year succeed in forming expectations for how objects will look in different orientations, despite young children's difficulties with verbal tests of mental rotation (see Frick et al., 2014). Moreover, infants succeed in representing others' false beliefs in nonverbal tests, despite young children's struggles with verbal false-belief tests (see Scott \& Baillargeon, 2017). However, it remains unknown whether infants represent others' differing perspectives on the same visible object. If infants have such an implicit understanding, then we may be able to detect it using nonverbal tasks, like those that have been developed to examine infants' understanding of what others can see (e.g., Luo \& Johnson, 2009). Infants' implicit understanding of different people's experiences of the same object may be especially clear when they are presented with upright and inverted human faces: pictures that elicit interest even in newborn infants, and whose orientation greatly affects face perception by human infants, children, and adults (e.g., Chien, 2011; Parr et al., 1998; Valenza et al., 1996; Van Der Geest et al., 2002). 


\section{Research Overview}

The present experiments focused on 14- to 15-month-old infants, because infants of this age have been shown to understand whether others can see objects, and in some studies, to represent others' false beliefs. We ask whether such infants can reason about the perspective of an actor who faced them. To test this understanding, we adapted prior work on infants' understanding of actions (that a person points or reaches for one object over another (Woodward, 1998; Woodward \& Guajardo, 2002) and tested whether infants represent the goal of that actor's action with respect to that actor's own perspective on objects on which he acts.

In all our experiments, the perspectives of the actor and of infants themselves were initially in direct conflict. In Experiment 1, pictures of faces that were visible to the actor were occluded to infants, and vice versa. In Experiments 2 and 3, pictures of faces that were upright to the actor were inverted to infants, and vice versa. To succeed in Experiment 1 , infants only needed to infer which faces the actor could and could not see. To succeed in Experiments 2 and 3, infants needed to infer which face the actor experienced as upright and which as upside down.

\section{Experiment 1}

In Experiment 1, we tested whether 14- to 15-month-old infants were sensitive to the visibility of pictures to an actor who was seated facing the infant. We familiarized infants to videos of an actor in front of two pictures of faces: one visible to the actor but occluded to infants by a screen, and one occluded to the actor by a second screen but visible to the infants (Fig. 1A). Because the actor faced each infant, his perspective on the faces was opposite that of the infant: What was visible to the actor was occluded to the infant, and the reverse.

On each familiarization trial, the actor pointed to faces that were visible (for half the infants) or occluded (for the other infants). In test trials, the actor moved to the near side of the room and turned around, such that his perspective and that of the infant now coincided. In alternating trials, he pointed in a manner that was consistent either with his own perspective during familiarization (Same-to-Actor trials), or with the infant's perspective during familiarization (Same-to-Baby trials).

If infants understand that the actor's perspective had changed, then they should expect the actor to point to the picture of the same visibility to the actor as in familiarization, looking longer in Same-to-Baby than in Same-to-Actor trials. If infants are instead egocentric, then they may expect the actor to point to the picture of the same visibility to themselves as in familiarization, looking longer in Same-toActor than Same-to-Baby trials. Finally, infants may see the actor's actions as an act of communication or pedagogy (especially because he is pointing), such that they may think that the actor is taking on infants' perspectives; if so, then they may also look longer in Same-to-Actor trials.

\section{Method}

Experiment 1's hypotheses, methods, and analysis plans were preregistered on the Open Science Framework. All preregistration documents and stimuli can be found at https://osf.io/ne32h/.

Participants Thirty-two 14- to 15-month-old infants (12 boys, 20 girls; mean age $=14.84$ months; range $=13 ; 17$ to $15 ; 27)$ contributed data to this experiment. We had preregistered this sample size based on a power analysis over pilot data. An additional infant began the experiment, but was not included in the final sample due to technical difficulties $(n=1)$. In all experiments, naïve experimenters determined exclusions using preset criteria.

Displays and procedure For all experiments, data collection took place over Zoom video calls, and infants sat in their parents' laps or on high chairs. Parents were instructed to sit quietly, not influence their infants, and look away from the displays in test trials. In Experiment 1, infants viewed displays either on laptops $(n=31)$, or a phone $(n=1)$.

In all experiments, there were 6 familiarization trials and 6 test trials. In Experiment 1, all trials depicted an actor in a room pointing to one of two pictures, one visible and one occluded to him. Events are outlined below (see Fig. 1A).

All familiarization trials began with two pictures of faces in a room, initially without an actor present. We then blocked each picture with a screen that occluded it on one side of the room. Then, an actor appeared on the far side of the room, facing forward, such that the picture visible to him was occluded to infants, and vice versa. The actor repeatedly pointed to pictures of a specific visibility to him (i.e., either always visible or always occluded). After pointing, a sound played to alert infants to the actor's action. The positions and identities of the visible and occluded pictures switched between familiarization trials. To reduce the likelihood that infants saw the actor's actions as communicative, the actor always looked down, and never looked back and forth between pictures and infants.

In all experiments, there were 6 pairs of faces: three used in familiarization, and three in test trials. Infants saw each pair of faces in one pair of trials. In Experiment 1's familiarization, the faces in each pair of trials alternated in being visible or occluded to the actor, and the identity of each face on the two sides of the actor remained constant. Because the actor always pointed to pictures of a specific visibility in familiarization, infants never saw the actor point to the same picture twice.

All test trials began with the actor moving from the far side of the room to the near side of the room, initially without pictures present. Before he turned around to face the center of the room, we placed pictures and dividers behind him. Then, the actor turned around, so that pictures that would have been visible to him from his position in familiarization were now occluded, and vice versa. In alternating test trials, the actor either pointed to each of the pictures. 


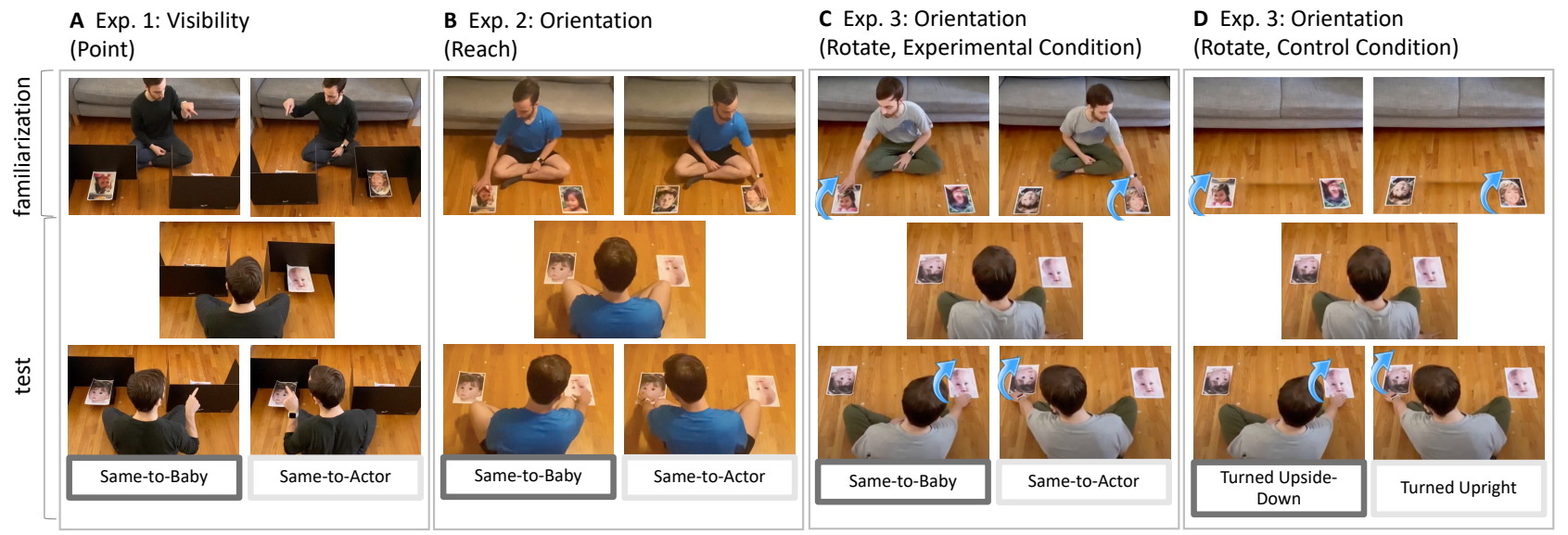

Figure 1. Still frames from stimuli. In Experiment 1's familiarization (A), an actor pointed to pictures of faces that were either always visible or occluded to him. In Experiment 1's test trials, the actor moved to the other side of the room, turned around, and then pointed to pictures that were of the same visibility, either to babies or to himself, as in familiarization. Experiment 2's familiarization (B), an actor reached to pictures that were either always upright or inverted to him. In Experiment 2's test trials, the actor moved to the near side of the room, turned around, and then reached for pictures that were in the same orientation, either to babies or to himself, as in familiarization. In familiarization for Experiment 3's Experimental Condition (C), an actor seated on the far side of a room reached for and rotated pictures that were inverted to him so that they became upright to him. In familiarization for Experiment 3's Control Condition (D), there was no actor, but the pictures rotated in the same way as in the Experimental Condition. In Experiment 3's test trials (which were the same for the two conditions), the actor moved to the near side of the room and turned around, and reached for pictures that were inverted to turn them upright, or for pictures that were upright to turn them inverted for himself. Blue curved arrows indicate that pictures are about to rotate $180^{\circ}$.

In both familiarization and test trials, after the actor pointed to a picture, the videos looped, and an experimenter (naïve to all events) began coding, using the coding program $\mathrm{jHab}$ (Casstevens, 2007). The coder coded looking time until the end of the trial, when infants had looked away for 2 consecutive seconds, or 30 total seconds had elapsed. A second coder recoded $25 \%$ of test trials offline in jHab, using the same thresholds. The intraclass correlation coefficient (ICC) between the two observers' data was 0.95 (95\% CI $[0.91,0.97])$.

Counterbalancing We counterbalanced: the visibility of pictures that the actor pointed to (i.e., his preferred visibility) in familiarization (visible/occluded to infants); the location of the picture that the actor pointed to in the first trial of each pair of familiarization trials (left/right to infants); and the order of Same-to-Actor trials in test (first/second). The location of the preferred-visibility picture in test trials was the same as the location of the preferred-visibility picture in the first trial of each pair of familiarization trials.

\section{Results}

Preregistered analysis All reported $p$-values are two-tailed. To determine whether infants looked longer to Same-toActor trials than Same-to-Baby trials, we examined looking times in a mixed-effects model. The dependent variable was looking time. The fixed effect was trial type (Same-toActor/Same-to-Baby). Participant ID was a random intercept. We found that infants looked longer during the Same-toBaby trials $\left(\right.$ mean $\left._{\text {same-to-baby }}=21.16 \mathrm{~s}\right)$ than during the Same-
to-Actor trials $\left(\right.$ mean $\left._{\text {same-to-actor }}=16.85 \mathrm{~s}\right)$ trials $(\beta=-0.48$, $95 \%$ of $\beta[-0.76,-0.21], b=-4.41, t(142)=-3.41, p<.001)$.

\section{Discussion}

In Experiment 1, 14- to 15-month-old infants looked longer in test trials when the actor acted consistently from the infants' perspective than when he acted consistently from his own perspective. These findings provide evidence that infants understood that the actor's action depended on his own perspective: When he had pointed to the pictures of faces that were occluded to him during familiarization, infants expected that he would point to the occluded faces in test, even though from the infants' perspective, the faces to which he pointed were previously visible to infants. Thus, infants were not egocentric. Moreover, these findings suggest that infants did not see the actor's pointing as an act of communication towards themselves (e.g., pointing to the face that the baby could not see to indicate that the face was present behind the screen), for if they had, then infants would have expected the actor to act consistently from the infants' perspective rather than his own perspective. Given infants' success in reasoning non-egocentrically about which faces the actor could see, we next ask, in a modified version of this task, whether infants can reason non-egocentrically about which faces another person experiences as upright or inverted.

\section{Experiment 2}

Experiment 2 used the same methods as Experiment 1, except as follows. We familiarized infants to videos of an actor 


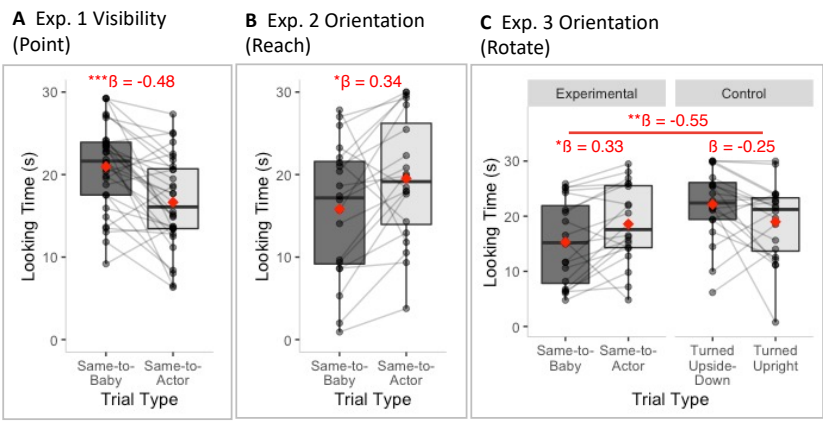

Figure 2. Results in Experiments 1 to 3, depicting the amount of time infants looked to different test trials. Red diamonds indicate means. Pairs of connected dots indicate data from a single infant. Horizontal lines within boxes indicate medians, and boxes indicate interquartile ranges. The beta coefficients indicate standardized effect sizes, following (in Experiment 2) corrections for multiple comparisons. Asterisks indicate significant differences $\left({ }^{*} p\right.$ $<.05,{ }^{* *} p<.01, * * * p<.001 ; 2$-tailed).

seated in front of the same pictures of faces, one upright and one inverted (Fig. 1B), without screens. In familiarization, the actor reached for faces in a specific orientation to him (i.e., always upright or always inverted). In test trials, the actor moved to the other side of the room and turned around, so that faces that were upright to him before were upsidedown to him now, and vice versa. In alternating trials, the actor either reached for faces that were in the same orientation to him as before (Same-to-Actor trials), or for faces that were in the same orientation to the infant as before (Same-to-Baby trials).

If infants understand that the actor's perspective has changed, then they should expect the actor to reach for faces in the same orientation to himself, looking longer in Sameto-Baby than in Same-to-Actor trials. By contrast, if infants are like young children tested verbally, and do not appreciate different perspectives on the same objects, then they should expect the actor to reach for faces that look to infants like the faces that he had reached to previously, looking longer in Same-to-Actor than in Same-to-Baby trials.

\section{Method}

Experiment 2's hypotheses, methods, and analysis plans were preregistered on the Open Science Framework and can be found at https://osf.io/ne32h/.

Participants We had preregistered a sample size of 16 based on a power analysis over pilot data. However, more parents responded than anticipated, resulting in a sample of twenty 14- to 15 -month-old infants (10 boys, 10 girls; mean age = 14.68 months; range $=13 ; 17$ to $15 ; 19$ ). An additional 2 infants began the experiment, but were not included in the final sample due to fussiness $(n=1)$ and technical difficulties $(n=1)$.

Displays and procedure Infants viewed displays either on laptops $(n=18)$, a TV $(n=1)$, or a phone $(n=1)$. All trials depicted an actor reaching for one of two faces, one upright and one inverted (Fig. 1B).

All familiarization displays and events were the same as in Experiment 1, except for the absence of occluders and the actor's action: He reached for faces in a specific orientation to him (i.e., either upright or inverted) instead of pointing. Upon contact with a face, a bell-like sound played. The positions and identities of the upright and inverted faces switched as in Experiment 1.

All test trials began with the actor moving from the far to the near side of the room and turning around, as in Experiment 1, so that what used to be upright to him in familiarization was now inverted, and vice versa. In alternating trials, the actor either reached for pictures that were in the same orientation to him (Same-to-Actor trials) or in the same orientation to the infants (Same-to-Baby trials).

As in Experiment 1, there were 6 pairs of different faces: three in familiarization, and three in test. In familiarization, the faces in each pair of trials rotated $180^{\circ}$ from the first trial to the second in the pair, and the identity of each face on the two sides of the actor remained constant. Because the actor always reached for faces in a specific orientation in familiarization, infants never saw the actor reach for the same face twice.

In both familiarization and test, after the bell-sound played, the videos looped, and a naïve experimenter began coding, using $\mathrm{jHab}$ (as in Experiment 1). A second coder recoded $25 \%$ of test trials offline in $\mathrm{jHab}$. The intraclass correlation coefficient (ICC) between the two observers' data was 0.98 (95\% CI [0.97, 0.99]).

Counterbalancing Counterbalancing was the same as in Experiment 1, except that it was based on the orientation of faces and on reaching.

\section{Results}

Preregistered analysis Infants' looking was analyzed using the same mixed-effects model as in Experiment 1. Infants looked longer to the Same-to-Actor test trials (mean same-to-actor $=18.84 \mathrm{~s}$ ) than to the Same-to-Baby test trials (mean same-to-baby $=16.05 \mathrm{~s})$ trials $(\beta=0.34,95 \% \mathrm{CI}$ of $\beta[0.07,0.62], b=3.46$, $t(66)=2.48, p=.015)$. This is the opposite effect to that obtained in Experiment 1.

\section{Discussion}

In Experiment 2, 14- to 15-month-old infants looked longer when the actor acted consistently from his own perspective than when he acted consistently from infants' perspective. These findings, which are opposite to the reasoning of older children and adults and to the pattern of findings in Experiment 1, suggest that infants were egocentric and did not appreciate that the actor's action would depend on his own distinctive perspective on the faces. In Experiment 3, we aimed to enhance infants' ability to appreciate another person's perspective by calling attention to the orientation of the faces during the familiarization period. 


\section{Experiment 3}

In Experiment 3, we asked whether infants better take account of a person's perspectives in our design if his action changes the orientation of a face, conveying to infants that his action is aimed at altering the face's orientation, and therefore altering his perspective on the object. Specifically, whereas the actor in Experiment 2 either had reached for pictures of faces that were upright or inverted to him in familiarization, in Experiment 3's Experimental Condition, the actor only reached for faces that were inverted to him in familiarization (and therefore upright to infants), and he rotated the faces $180^{\circ}$ so that they became upright to him (and inverted to infants) (Fig. 1C). This rotation served two purposes. First, it highlighted the orientation of the faces from the actor's perspective for infants, who typically prefer to look at upright faces (e.g., Valenza et al., 1996), and may expect others to share this preference. Second, this rotation may allow infants to better appreciate that the upright faces that they see become inverted when rotated $180^{\circ}$.

In the Experimental Condition's test trials, the actor moved to the other side of the room and alternately reached for faces that were inverted to him and rotated them (Same-to-Actor trials), or reached for faces that were upright to him and rotated them (Same-to-Baby trials). If babies do not distinguish differing perspectives on the same face, even when perspectives on that face change during each trial, then they should again look longer in Same-to-Actor trials, when the actor reaches for and rotates faces so that they become upright to him in test trials.

We recognized, however, that the interpretation of looking patterns during the test trials could be complicated by an intrinsic preference for faces that are made upright, as would happen in Experiment 3's Same-to-Actor trials. Moreover, there are low-level perceptual differences between familiarization and test in the Experimental Condition: At test, babies saw two upright faces simultaneously for the first time. To address these confounds, we tested one group of infants in the Experimental Condition just described, and a second group of infants in a Control Condition, in which only the pictures were present during the familiarization trials and they appeared to rotate by themselves. The Control Condition's test trials were the same as those of the Experimental Condition, enabling us to distinguish responses in the Experimental Condition that depend on attributions of perspectives to the actor from responses that depend on preferences for upright faces or for visually novel events.

\section{Method}

Experiment 3's hypotheses, methods, and analysis plans were preregistered on the Open Science Framework. All preregistrations and stimuli can be found at https://osf.io/ne32h/.

Participants Forty 14- to 15-month-old infants contributed data ( 22 boys, 18 girls; mean age $=15 ; 10$ months; range $=$ $14 ; 2$ to $15 ; 29)$. None of the infants met our exclusion criteria. We had preregistered this sample size based on power analyses over pilot data and data from Experiment 2.

Displays Infants viewed displays either on laptops $(n=33)$, a desktop computer $(n=4)$, a tablet $(n=2)$, or a desktop computer $(n=1)$.

Infants were assigned to either the Experimental Condition $(n=20)$ or the Control Condition $(n=20)$. Events are outlined below (see Fig. 1C).

All familiarization trials of the Experimental Condition began with the actor on the far side of the room, facing infants and looking down. The actor repeatedly reached for pictures that were inverted to him (and upright to babies, who did not share his perspective), and rotated them $180^{\circ}$ so that they became upright to him (and inverted to babies). The positions and identities of the upright and inverted pictures switched between familiarization trials, and the actor always reached for and rotated upside-down pictures.

The familiarization trials of the Control Condition were like those of the Experimental Condition in timing and in sound, but the actor was not present in the scene. Instead, the screen faded to black and a bell-like sound played during the time when the actor in the Experimental Condition would have acted. Then the room became visible again, revealing that one of the pictures (the one that was initially upright to babies) was now inverted to the infants. Thus, infants in the Experimental and Control Conditions saw the same pictures, in the same orientations, to control for any potential effect of perceptual familiarity of the face displays at test.

Test trials followed the procedure of Experiments 1 and 2. In the Experimental Condition, the actor reached for and rotated pictures that were either in the same orientation to him (Same-to-Actor) or to the baby (Same-to-Baby). In the Control Condition, because infants did not see the actor act in familiarization, we instead refer to these test trials by the final orientation of the pictures in the trials for both infants and for the actor: Turned Upright and Turned Upside-Down trials.

As in Experiments 1 and 2, there were 6 pairs of different faces. Infants saw each pair of faces in one pair of trials, and faces rotated from the first to the second familiarization trial in a pair as in Experiment 2.

In both familiarization and test, after the bell-sound played, the videos looped, and a naïve experimenter began coding, using $\mathrm{jHab}$ (as in Experiment 1). A second coder recoded $25 \%$ of test trials offline in $\mathrm{jHab}$. The intraclass correlation coefficient (ICC) between the two observers' data was 0.98 (95\% CI [0.97, 0.98]).

Counterbalancing Counterbalancing was the same as in Experiment 2, except that there was no reaching in familiarization of the Control Condition.

\section{Results}

Preregistered analysis To determine whether looking times differed for test trials in the two conditions, we ran a mixedeffects model like that of our other experiments, but with 
different fixed effects: trial type according to the infants' perspective in test (Turned Upright/Turned Upside-Down), condition (Experimental/Control), and the interaction; these fixed effects were centered. (For this model, given that the videos in test trials were the same between conditions, we included Same-to-Actor trials as Turned Upright trials, and Same-to-Baby trials as Turned Upside-Down trials.).

There was a significant interaction $(\beta=-0.55,95 \% \mathrm{CI}$ of $\beta$ $[-1.03,-0.14], b=-5.55, t(147)=-2.89, p=.004)$. Posthoc pairwise tests, correcting for multiple comparisons using Holm's method, revealed that infants looked longer to Sameto-Actor $\left(\right.$ mean $_{\text {same-to-actor }}=18.87 \mathrm{~s}$ ) than to Same-to-Baby $\left(\right.$ mean $\left._{\text {same-to-baby }}=15.40 \mathrm{~s}\right)$ trials in the Experimental Condition $(\beta=0.33, b=3.26, t(112)=2.39, p=.018)$, but that infants did not look differently to the same videos in the Control Condition $(\beta=-0.25, b=-2.29, t(114)=-1.66, p=.099)$, not looking differently in Turned Upside-Down (mean upside-down $=$ $22.32 \mathrm{~s})$ and Turned Upright (mean upright $=19.91 \mathrm{~s})$ trials.

\section{Discussion}

As in Experiment 2, 14- to 15-month-old infants in Experiment 3's Experimental Condition looked longer in test trials when the actor acted consistently from his own perspective than when he acted consistently from infants' perspective. The pattern of findings in our Control Condition provides evidence that infants' looking times in the Experimental Condition cannot be explained by a preference for looking at upright pictures, or by low-level perceptual differences between familiarization and test trials.

If anything, there was a qualitative trend in the Control Condition for infants to look longer when the actor rotated pictures so that they become inverted in test. This trend would be consistent with the possibility that infants, who themselves have an early emerging preference for upright over inverted faces (e.g., Valenza et al., 1996), may expect that others will hold those preferences. By contrast, when infants in the Experimental Condition were familiarized to videos of the actor rotating pictures so that they became upright to the actor (but inverted to infants), they showed the opposite looking pattern. This pattern of findings suggests that infants were sensitive to the actor's reaching in familiarization, but did not take into account that his perspective differed from their own.

\section{Interpreting Looking Times}

Throughout our experiments, we have assumed that longer looking indicates a response to novelty, which is consistent with past studies on mental state reasoning in infants that have similarly familiarized infants to an agent acting on objects (e.g., Luo \& Johnson, 2009; Onishi \& Baillargeon, 2005). Following this assumption, we have argued: (i) that the pattern of infants' looking in Experiment 1 provides evidence that they inferred that they and the actor could not see the same pictures in familiarization; and (ii) that the opposite pattern of infants' looking in Experiment 2 and Experiment 3's Experimental Condition provides evidence that infants failed to infer that they and the actor experienced pictures of faces in different orientations in familiarization.
In some studies, however, infants look longer to familiar displays if they are merely familiarized (rather than habituated) to complex events before the test phase (e.g., Hunter et al., 1983). This pattern suggests an alternative account of our findings: If representing others' differing perspectives on the same object (Experiments 2 and 3) is substantially more complex than representing whether others see objects (Experiment 1), then infants who are only familiarized could look longer to novel events in Experiment 1, but look longer to familiar events in Experiments 2 and 3. That is, task complexity may alter the direction of infants' looking preferences. Importantly, this alternative account would predict that infants who had habituated in Experiments 2 and 3 should be more likely to exhibit novelty preferences.

We ran an exploratory analysis to determine whether infants' looking times in test trials for Experiment $2(n=20)$ and Experiment 3's Experimental Condition $(n=20)$ differed as a function of infants' habituation status. We categorized infants as having habituated if their total looking time in the last 3 familiarization trials was less than half that of the first 3 familiarization trials: the standard criterion of habituation. Of the 40 infants, 17 had habituated.

We ran an additional mixed-effects model, with the added fixed effect of habituation status (yes/no) and the interaction of habituation status and trial type (Same-to-Baby/Same-toActor). Fixed effects were centered. The interaction was not significant $(\beta=-0.28, b=-2.85, t(173)=-1.21, p=.220)$, suggestive that infants responses to test trials did not differ if they had habituated. Of the 17 infants who habituated, moreover, a significant majority (76\%) looked longer in Same-to-Actor than in Same-to-Baby trials (binomial $p=$ .049 ; bootstrapped $95 \%$ CI $[58 \%, 88 \%]$ ), indicating that infants viewed the test action of reaching in accord with the actor's perspective to be more novel. We therefore stand by our interpretation that infants expected the actor to act in accord with infants' perspective, rather than in accord with the actor's own perspective.

\section{General Discussion}

In human social life, we must understand not only whether others can see particular objects or events, but others' experiences of those objects and events. Human conversation often revolves around our differing experiences of the same dinner, the same music, the same political speech. In our experiments, we found that infants appreciated that two faces that were visible and occluded to themselves were instead occluded and visible, respectively, to an actor facing infants (Experiment 1), but that infants were egocentric when reasoning about others' perspectives on two faces that were both visible to infants and an actor (Experiments 2 and 3). While Experiment 1 confirms past findings that infants can reason about whether an object is visible to someone whose perspective differs from infants' own, Experiments 2 and 3 are the first to test infants' understanding of others' differing perspectives on the same visible object.

Our findings point to a level of mental state reasoning that infants may not yet be capable of, and that remains untested 
in nonhuman primates and other animals: an appreciation that the same visible object may be experienced differently (as upright versus inverted) by the self and others, depending on one's perspective. Our experiments introduce a method for testing this capacity in nonverbal populations; this method could be adapted to probe the evolutionary origins of this ability in other species.

If this ability is not present in infancy, then future research will be critical to understand what causes it to emerge by 4 years of age. We are now testing whether language plays a role in helping infants to appreciate how others' perspectives differ from their own. We also are testing whether infants are more sensitive to others' perspectives in social contexts, rather than when acting on inanimate pictures, as in the present studies (see Woo \& Spelke, 2020).

The ability to reason about others' differing perspectives is central to human social interaction. Whereas past research on infants has demonstrated impressive abilities for determining whether an object to another agent, the present experiments suggest that 14- to 15-month-old infants are egocentric when reasoning about different agents' experiences of the same visible objects. These experiments raise the bar for testing infants' representations of others' perspectives, suggest a limit to infants' mental state reasoning, and call on future research to probe both the nature and sources of this limit and the process by which it is overcome.

\section{Acknowledgements}

We thank the families who participated; Bill Pepe and Lauren Salmans for research assistance; Michael Gajda for acting in stimuli; and Hyowon Gweon and the Stanford Social Learning Lab for sharing protocols to facilitate online testing. This material is based on work supported by the Center for Brains, Minds, and Machines, funded by National Science Foundation STC Award CCF-1231216; by DARPA Award CW3013552; and by a Social Sciences and Humanities Research Council Doctoral Fellowship under award 752-2020-0474.

\section{References}

Birch, S. A., \& Bloom, P. (2004). Understanding children's and adults' limitations in mental state reasoning. Trends in Cognitive Sciences, 8(6), 255-260.

Casstevens, R. M. (2007). jHab: Java habituation software (version 1.0. 2) [computer software]. Chevy Chase, MD.

Chien, S. H.-L. (2011a). No more top-heavy bias: Infants and adults prefer upright faces but not top-heavy geometric or face-like patterns. Journal of Vision, 11(6), 13-13.

Flavell, J. H., Everett, B. A., Croft, K., \& Flavell, E. R. (1981). Young children's knowledge about visual perception: Further evidence for the Level 1-Level 2 distinction. Developmental Psychology, 17(1), 99-103.

Flavell, J. H., Shipstead, S. G., \& Croft, K. (1978). Young children's knowledge about visual perception: Hiding objects from others. Child Development, 1208-1211.
Frick, A., Möhring, W., \& Newcombe, N. S. (2014). Development of mental transformation abilities. Trends in Cognitive Sciences, 18(10), 536-54

Hamlin, J. K., Ullman, T., Tenenbaum, J., Goodman, N., \& Baker, C. (2013). The mentalistic basis of core social cognition. Developmental Science, 16(2), 209-226.

Hunter, M. A., Ames, E. W., \& Koopman, R. (1983). Effects of stimulus complexity and familiarization time on infant preferences for novel and familiar stimuli. Developmental Psychology, 19(3), 338-352.

Luo, Y., \& Johnson, S. C. (2009). Recognizing the role of perception in action at 6 months. Developmental Science, 12(1), 142-149.

Masangkay, Z. S., McCluskey, K. A., McIntyre, C. W., SimsKnight, J., Vaughn, B. E., \& Flavell, J. H. (1974). The early development of inferences about the visual percepts of others. Child Development, 357-366.

Onishi, K. H., \& Baillargeon, R. (2005). Do 15-month-old infants understand false beliefs? Science, 308(5719), 255258.

Phillips, J., Buckwalter, W., Cushman, F., Friedman, O., Martin, A., Turri, J., Santos, L., \& Knobe, J. (undefined/ed). Knowledge before belief. Behavioral and Brain Sciences, 1-37.

Scott, R. M., \& Baillargeon, R. (2017). Early false-belief understanding. Trends in Cognitive Sciences, 21(4), 237249.

Southgate, V., Senju, A., \& Csibra, G. (2007). Action anticipation through attribution of false belief by 2 -yearolds. Psychological Science, 18(7), 587-592.

Surtees, A. D. R., Butterfill, S. A., \& Apperly, I. A. (2012). Direct and indirect measures of Level-2 perspective-taking in children and adults. British Journal of Developmental Psychology, 30(1), 75-86.

Valenza, E., Simion, F., Cassia, V. M., \& Umiltà, C. (1996). Face preference at birth. Journal of Experimental Psychology: Human Perception and Performance, 22(4), 892-903.

Van Der Geest, J. N., Kemner, C., Verbaten, M. N., \& Engeland, H. V. (2002). Gaze behavior of children with pervasive developmental disorder toward human faces: A fixation time study. Journal of Child Psychology and Psychiatry, 43(5), 669-678.

Woo, B. M., \& Spelke, E. (2020). Infants' social evaluations depend on the intentions of agents who act on false beliefs. PsyArXiv. https://https://doi.org/10.31234/osf.io/eczgp

Woo, B. M., Steckler, C. M., Le, D. T., \& Hamlin, J. K. (2017). Social evaluation of intentional, truly accidental, and negligently accidental helpers and harmers by $10-$ month-old infants. Cognition, 168, 154-163.

Woodward, A. L. (1998). Infants selectively encode the goal object of an actor's reach. Cognition, 69(1), 1-34.

Woodward, A. L., \& Guajardo, J. J. (2002). Infants' understanding of the point gesture as an object-directed action. Cognitive Development, 17(1), 1061-1084. 